\title{
Meridional flow of grounded abyssal currents on a sloping bottom in spherical geometry
}

\author{
G. E. Swaters \\ Applied Mathematics Institute, Department of Mathematical \\ \& Statistical Sciences and Institute for Geophysical Research, \\ University of Alberta, Canada
}

\begin{abstract}
A steady nonlinear planetary-geostrophic model in spherical coordinates is presented describing the hemispheric-scale meridional flow of grounded abyssal currents on a sloping bottom. The model, which corresponds mathematically to a quasi-linear hyperbolic partial differential equation, can be solved explicitly for a cross-slope isopycnal field that is grounded (i.e., intersects the bottom on the up slope and down slope sides). The abyssal currents possess decreasing thickness in the equatorward direction while maintaining constant meridional volume transport and exhibit westward intensification as they flow toward the equator.
\end{abstract}

\section{Introduction}

Many of the abyssal currents in the oceans associated with the equatorward motion of deep water masses produced in high latitudes due to atmospheric cooling, are organized as mesoscale topographically-steered geostrophicallybalanced grounded gravity currents that flow along sloping continental boundaries. These currents form an important component in the deep "leg" of the meridional overturning circulation in the oceans. The mesoscale dynamics of these currents has been described in a series of papers [1-11]. All of these studies have implicitly assumed either an $f$ or $\beta$-plane approximation in which the dynamics is modelled in a Cartesian coordinate system with the implicit assumption that the horizontal length scales are not too much larger than the internal deformation radius (on the order of about 10-100 km in the ocean). 
However, the spatial extent of these abyssal currents is well known to be hemispheric in scale. This raises the question about the role of planetary sphericity in determining the large scale kinematic structure of these flows.

The principal purpose of this paper is to present a preliminary report on a simple, but nevertheless illuminating, reduced-gravity shallow-water model for the meridional, or equatorward, steady flow of grounded abyssal currents on a longitudinally-sloping bottom on a rotating sphere. A complete discussion of the dynamical properties as well as a specific oceanographically-relevant example of the solution briefly described here will be published elsewhere in a venue more suitable for research problems in geophysical fluid dynamics.

\section{Model derivation}

The reduced-gravity shallow-water equations for a stably-stratified abyssal water mass overlying variable bottom topography on a rotating sphere can be written in the form

$$
\begin{gathered}
u_{t}+\frac{u u_{\lambda}}{a \cos \theta}+\frac{v u_{\theta}}{a}-\frac{u v \tan \theta}{a}-2 \Omega v \sin \theta \\
=-\frac{g^{\prime}}{a \cos \theta}\left(h+h_{B}\right)_{\lambda}, \\
v_{t}+\frac{u v_{\lambda}}{a \cos \theta}+\frac{v v_{\theta}}{a}-\frac{v^{2} \tan \theta}{a}+2 \Omega u \sin \theta=-\frac{g^{\prime}}{a} h_{\theta}, \\
h_{t}+\frac{1}{a \cos \theta}\left[(h u)_{\lambda}+(h v \cos \theta)_{\theta}\right]=0,
\end{gathered}
$$

where $u$ and $v$ are the zonal (positive eastward) and meridional (positive northward) velocities, respectively, $\lambda$ is the longitude (positive eastward) and $\theta$ is the latitude (positive northward), $t$ is time, $\Omega$ is the angular frequency associated with Earth's rotation ( $2 \pi$ rads/day), $a$ is the radius of Earth (about $6300 \mathrm{~km}$ ), $h(\lambda, \theta, t)$ is the thickness of the abyssal layer, $h_{B}(\lambda)$ is the longitudinallyvarying height of the bottom topography above a constant reference depth and $g^{\prime}=g\left(\rho_{2}-\rho_{1}\right) / \rho_{2}>0$ is the (stably-stratified) reduced gravity where $\rho_{1}$ and $\rho_{2}$ are the densities of the (infinitely deep and motionless) overlying fluid and dynamically active abyssal layer, respectively, and $g$ is the gravitational acceleration $\left(9.81 \mathrm{~m} / \mathrm{s}^{2}\right)$. Typical oceanographic values for the reduced gravity are in the range $10^{-4}-10^{-2} \mathrm{~m} / \mathrm{s}^{2}$. The dynamic pressure (i.e., the total pressure minus the hydrostatic pressure) in the abyssal layer is given by $p=g^{\prime} \rho_{1}\left(h+h_{B}\right)$.

Further analysis is facilitated by introducing the scalings

$$
\begin{gathered}
\lambda=\frac{L}{a} \widetilde{\lambda}, t=\frac{a}{V} \widetilde{t}, u=\frac{L V}{a} \widetilde{u}, v=V \widetilde{v}, \\
\left(h, h_{B}\right)=\frac{2 \Omega V L}{g^{\prime}}\left(\widetilde{h}, \widetilde{h}_{B}\right), p=2 \Omega V L \rho_{1} \widetilde{p},
\end{gathered}
$$


into (1)-(3), yielding (after dropping the tildes)

$$
\begin{gathered}
\varepsilon \delta^{2}\left[u_{t}+\frac{u u_{\lambda}}{\cos \theta}+v u_{\theta}-u v \tan \theta\right]-v \sin \theta= \\
-\frac{1}{\cos \theta}\left(h+h_{B}\right)_{\lambda}, \\
\varepsilon\left[v_{t}+\frac{u v_{\lambda}}{\cos \theta}+v v_{\theta}-v^{2} \tan \theta\right]+u \sin \theta=-h_{\theta}, \\
h_{t}+\frac{1}{\cos \theta}\left[(h u)_{\lambda}+(h v \cos \theta)_{\theta}\right]=0,
\end{gathered}
$$

where $\varepsilon$ and $\delta$ are, respectively, the Rossby number and aspect ratio given by

$$
\varepsilon=\frac{V}{2 \Omega L} \text { and } \delta=\frac{L}{a},
$$

and where the dynamic pressure is given by

$$
p=h+h_{B} .
$$

Assuming typical scales [12] of

$$
V \simeq 5 \times 10^{-2} \mathrm{~m} / \mathrm{s}, L \simeq 10^{5} \mathrm{~m} \text { and } g^{\prime} \simeq 3 \times 10^{-3} \mathrm{~m} / \mathrm{s}^{2},
$$

suggests that

$$
\varepsilon \simeq 3.4 \times 10^{-4} \text { and } \delta \simeq 1.6 \times 10^{-2},
$$

with the additional scalings

$$
\frac{a}{V} \simeq 145 \text { days, } \frac{L V}{a} \simeq 8 \times 10^{-4} \mathrm{~m} / \mathrm{s} \text { and } \frac{2 \Omega V L}{g^{\prime}} \simeq 240 \mathrm{~m} .
$$

Thus, to leading order in the Rossby number, the model reduces to

$$
\begin{gathered}
u=-\frac{1}{\sin \theta} h_{\theta}, \\
v=\frac{1}{\sin \theta \cos \theta}\left(h+h_{B}\right)_{\lambda}, \\
\sin ^{2} \theta h_{t}+\tan \theta h_{B_{\lambda}} h_{\theta}-h h_{\lambda}=h_{B_{\lambda}} h .
\end{gathered}
$$

Equations (8)-(10) corresponds to a planetary-geostrophic model in which the velocities are geostrophically determined but for which order-one dynamic variations in the thickness in the abyssal layer are permitted, i.e., the thickness field can intersect the bottom allowing for groundings. It is important to point out that the model is singular at the equator $(\theta=0)$ and thus cannot be used to described inter-hemispheric or cross-equatorial flow. The dynamic pressure $p=h+h_{B}$ forms the geostrophic stream function for the flow as seen in (8) and (9). 


\section{Steady state solution}

We briefly describe the equilibrium solution to the model (8)-(10) in which $h(\lambda, \theta)$ will be determined by the steady-state quasi-linear hyperbolic equation

$$
\tan \theta h_{\theta}-\frac{h}{h_{B_{\lambda}}} h_{\lambda}=h .
$$

The model (11) can be solved using the method of characteristics with the boundary condition

$$
h\left(\lambda, \theta_{0}\right)=h_{0}(\lambda),
$$

where $\theta_{0}$ is a given reference latitude and $h_{0}(\lambda)$ is a prescribed abyssal height profile that varies only in the latitudinal direction.

The solution to (11) subject to (12) can be written in the form

$$
\begin{gathered}
h(\lambda, \theta)=\frac{\sin \theta}{\sin \theta_{0}} h_{0}(\tau), \\
h_{B}(\tau)+\frac{\sin \theta_{0}-\sin \theta}{\sin \theta_{0}} h_{0}(\tau)=h_{B}(\lambda) .
\end{gathered}
$$

Given $\lambda$ and $\theta$, one solves (14) for $\tau(\lambda, \theta)$ and then determines $h(\lambda, \theta)$ from (13).

\section{Some properties of the solution}

The first thing to note is that the abyssal height monotonically decreases in the equatorward direction and in the limit as $\theta \rightarrow 0$, it follows from (13) that $h(\lambda, \theta) \rightarrow 0$.

The characteristics associated with the quasi-linear model (11) are the curves in $(\lambda, \theta)$-space along which $\tau$ is constant, as determined by (14). Along these characteristic curves

$$
\left.\frac{d \theta}{d \lambda}\right|_{\tau=\text { constant }}=-\frac{h_{B}^{\prime}(\lambda) \sin \theta_{0}}{\cos \theta h_{0}(\tau)} .
$$

We are interested in the physical situation where $h_{B}^{\prime}(\lambda)<0$ with $\theta>0$ corresponding to flow in the northern hemisphere along a topographic slope in which the depth of the fluid increases as $\lambda$ increases, i.e., eastward. This is a model for the equatorward flow of a grounded abyssal water masses along a continental slope on the western side of an ocean basin. In this situation the characteristics are, generally speaking, aligned in the southwest to the northeast direction since

$$
-\frac{h_{B}^{\prime}(\lambda) \sin \theta_{0}}{\cos \theta h_{0}(\tau)}>0 .
$$

In fact, the characteristics correspond to the geostrophic streamlines. Equations (13) and (14) can be combined into the form

$$
p(\lambda, \theta) \equiv h_{B}(\lambda)+h(\lambda, \theta)=h_{B}(\tau)+h_{0}(\tau) .
$$


Thus, when $\tau$ is constant, i.e., along a characteristic, the geostrophic stream function is constant and the characteristics coincide with the streamlines. The characteristics, therefore, describe the path lines in the flow and given the orientation of the characteristics associated with (15) when $h_{B}^{\prime}(\lambda)<0$ with $\theta>0$, the equatorward flow is, generally speaking, moving in the northeast to the southwest direction as it is, nevertheless, topographically steered.

The corresponding velocity components, determined by (8) and (9) are given by, respectively

$$
\begin{gathered}
u(\lambda, \theta)=-\frac{\left[h_{B}^{\prime}(\tau)+h_{0}^{\prime}(\tau)\right] \tau_{\theta}}{\sin \theta} \\
=-\frac{\cot \theta h_{0}(\tau)\left[h_{B}^{\prime}(\tau)+h_{0}^{\prime}(\tau)\right]}{\sin \theta_{0} h_{B}^{\prime}(\tau)+\left(\sin \theta_{0}-\sin \theta\right) h_{0}^{\prime}(\tau)}, \\
v(\lambda, \theta)=\frac{\left[h_{B}^{\prime}(\tau)+h_{0}^{\prime}(\tau)\right] \tau_{\lambda}}{\sin \theta \cos \theta} \\
=\frac{\sin \theta_{0} h_{B}^{\prime}(\tau)\left[h_{B}^{\prime}(\tau)+h_{0}^{\prime}(\tau)\right]}{\sin \theta \cos \theta\left[\sin \theta_{0} h_{B}^{\prime}(\tau)+\left(\sin \theta_{0}-\sin \theta\right) h_{0}^{\prime}(\tau)\right]}
\end{gathered}
$$

where (14) and (16) have been used. Observe that as $\theta \rightarrow 0$, both $u$ and $v$ become unbounded as a consequence of the fact that the geostrophic balance ((8) and (9)) breaks down at the equator. The meridional and zonal mass fluxes given by $v h$ and $u h$, respectively, remain bounded over the entire domain (including at the equator).

Another general property of the solution is that the position of the groundings in the abyssal height field, that is, the location(s) where $h(\lambda, \theta)=0$, i.e., $h$ intersects the bottom, are invariant with respect to $\theta$ once set by their location in the boundary condition (12). This means that throughout the domain the groundings will simply correspond to the fixed $\lambda$-values for which $h_{0}(\lambda)=0$. To see this suppose that a grounding in the solution occurs along the curve $\lambda=\widetilde{\lambda}(\theta)$ (allowing for a possible $\theta$-dependence). It follows from (13) that

$$
\begin{gathered}
h(\tilde{\lambda}(\theta), \theta)=0=\frac{\sin \theta}{\sin \theta_{0}} h_{0}(\tau(\widetilde{\lambda}(\theta), \theta)) \\
\Longrightarrow h_{0}(\tau(\tilde{\lambda}(\theta), \theta))=0 .
\end{gathered}
$$

But it therefore follows from (14) that

$$
h_{B}(\tau(\tilde{\lambda}(\theta), \theta))=h_{B}(\tilde{\lambda}(\theta)) \Longrightarrow \tau(\tilde{\lambda}, \theta)=\tilde{\lambda},
$$

since $h_{0}(\tau(\tilde{\lambda}, \theta))=0$. Thus, along a grounding we necessarily have $\lambda=\widetilde{\lambda}$ where $h_{0}(\widetilde{\lambda})=0$, i.e., the $\lambda$-location of a grounding is independent of $\theta$ and is set by the boundary data along $\theta=\theta_{0}$.

The fact that the meridional location of the groundings does not vary with latitude $\theta$, together with the fact as previously established, that the streamlines are oriented in the northeast to the southwest direction (when $h_{B}^{\prime}(\lambda)<0$ with 
$\theta>0)$ means that the flow exhibits a westward intensification, i.e., as the flow moves equatorward the streamlines shift westward or in the onshore or up slope direction. In fact, as shown below, it is possible, depending on the initial conditions for a "shock" to form in the solution on the western or up slope or western flank. The formation of such a shock could result in mixing on the up slope flank of the abyssal water mass as it propagates equatorward. This would be a decidedly different "instability mechanism" than baroclinic destabilization, which preferentially occurs on the down slope or offshore side of grounded abyssal currents $[7,8,10]$.

The net meridional volume transport is also constant with respect to $\theta$. Suppose that the abyssal current height $h_{0}(\lambda)$ is only nonzero in the region $\lambda_{1}<\lambda<\lambda_{2}$, i.e., $h_{0}\left(\lambda_{1,2}\right)>0$ only for $\lambda \in\left(\lambda_{1}, \lambda_{2}\right)$ with $h_{0}\left(\lambda_{1,2}\right)=0$. The net meridional volume transport as a function of $\theta$ is given by

$$
\begin{gathered}
T \equiv \int_{\lambda_{1}}^{\lambda_{2}} h(\lambda, \theta) v(\lambda, \theta) \cos (\theta) d \lambda \\
=\int_{\lambda_{1}}^{\lambda_{2}} \frac{h\left(h+h_{B}\right)_{\lambda}}{\sin (\theta)} d \lambda \\
=\frac{1}{\sin \theta_{0}} \int_{\lambda_{1}}^{\lambda_{2}} h_{0}(\tau)\left[h_{0}(\tau)+h_{B}(\tau)\right]_{\lambda} d \lambda \\
=\frac{1}{\sin \theta_{0}} \int_{\lambda_{1}}^{\lambda_{2}} h_{0}(\tau)\left[h_{0}^{\prime}(\tau)+h_{B}^{\prime}(\tau)\right] d \tau \\
=\frac{1}{\sin \theta_{0}} \int_{\lambda_{1}}^{\lambda_{2}} h_{0}(\tau) h_{B}^{\prime}(\tau) d \tau,
\end{gathered}
$$

which is independent of $\theta$, where (13), (14) and (19) have been used.

As discussed previously, the westward intensification of the streamlines or characteristics as the flow moves equatorward (when $h_{B}^{\prime}(\lambda)<0$ with $\theta>0$ ) could result in shock formation on the western flank of the propagating abyssal water mass. The formation of a shock in the solution will correspond to the first $\theta$-value (as $\theta$ decreases from $\theta_{0}>0$ ) where $\left|h_{\lambda}\right| \rightarrow \infty$ (assuming flow in the northern hemisphere with $h_{B}^{\prime}(\lambda)<0$ ). From (13) it follows that

$$
\begin{gathered}
h_{\lambda}=\frac{\sin \theta}{\sin \theta_{0}} h_{0}^{\prime}(\tau) \tau_{\lambda} \\
=\frac{\sin \theta h_{0}^{\prime}(\tau) h_{B}^{\prime}(\tau)}{\sin \theta_{0} h_{B}^{\prime}(\tau)+\left(\sin \theta_{0}-\sin \theta\right) h_{0}^{\prime}(\tau)},
\end{gathered}
$$

where (14) has been used. Thus, the shock will occur for the first value of $\theta \leq \theta_{0}$ for which

$$
\sin \theta=\left[1+\frac{h_{B}^{\prime}(\tau)}{h_{0}^{\prime}(\tau)}\right] \sin \theta_{0},
$$


which we denote by $\theta_{s}$, and is given by

$$
\sin \theta_{s}=\left[1+\max _{\tau}\left(\frac{h_{B}^{\prime}(\tau)}{h_{0}^{\prime}(\tau)} \mid h_{0}^{\prime}(\tau)>0\right)\right] \sin \theta_{0},
$$

where we assume $h_{B}^{\prime}(\tau)<0$. Should it be the case that

$$
\max _{\tau}\left(\frac{h_{B}^{\prime}(\tau)}{h_{0}^{\prime}(\tau)} \mid h_{0}^{\prime}(\tau)>0\right)<-1,
$$

then $\theta_{s}<0$ and this is not physically relevant since the solution is singular at the equator $\theta=0$ in any event and cannot be extended into the southern hemisphere where $\theta<0$. Thus, the only physically relevant case is when

$$
-1<\max _{\tau}\left(\frac{h_{B}^{\prime}(\tau)}{h_{0}^{\prime}(\tau)} \mid h_{0}^{\prime}(\tau)>0\right)<0 .
$$

In only this case is it possible that a shock forms on the up slope or western flank of the equatorward propagating abyssal water mass (in the northern hemisphere) and the potential for mixing by nonbaroclinic instability processes can develop. This is an interesting idea that requires further study.

\section{Summary}

A preliminary report on a simple, but nevertheless illuminating, reduced-gravity shallow-water model for the meridional, or equatorward, flow of grounded abyssal currents on a longitudinally-sloping bottom on a rotating sphere has been given. For oceanographically relevant parameter values it was shown that the abyssal layer height satisfies a quasi-linear hyperbolic equation with the velocities being geostrophically determined. The model corresponds to a planetary-geostrophic dynamical balance that permits the abyssal height field to intersect the bottom, i.e., a so-called grounding. Due to the fact that model is singular at the equator, it cannot be used to investigate cross-equatorial or inter-hemispheric flow.

The steady-state limit of the equation governing the abyssal layer height could be solved exactly. For a physical configuration corresponding to flow in the northern hemisphere on topography associated with increasing ocean depth in the eastward direction, the abyssal current flows equatorward from the northeast to southwest direction. The characteristics associated with the quasi-linear hyperbolic model were shown to be co-parallel with the geostrophic streamlines. It was shown that the groundings could not vary with latitude and are therefore set by the northern boundary condition. The meridional volume transport was shown to be independent of latitude. Finally, conditions for possible shock formation in the solution were established. If a shock forms, it is possible that this could lead to mixing on the up slope or western flank of these propagating abyssal water masses, which would be quite different than the instability and mixing associated with baroclinic destabilization. These and other issues require further study. 


\section{Acknowledgement}

Preparation of this paper was partially supported by the Natural Sciences and Engineering Research Council of Canada.

\section{References}

[1] Choboter, P. F. \& Swaters, G. E., Two layer models of abyssal equator crossing flow. Journal of Physical Oceanography, 33, pp. 1401-1415, 2003.

[2] Choboter, P. F. \& Swaters, G. E., Shallow water modeling of Antarctic Bottom Water crossing the equator. Journal of Geophysical Research, 109, C03038, doi:10.1029/2003JC002048, 2004.

[3] Ha, S. J. \& Swaters, G. E., Finite amplitude baroclinic instability of time varying abyssal currents. Journal of Physical Oceanography, 36, pp. 122 $139,2005$.

[4] Mooney, C. J. \& Swaters, G. E., Finite amplitude baroclinic instability of a mesoscale gravity current in a channel. Geophysical and Astrophysical Fluid Dynamics, 82, pp. 173-205, 1996.

[5] Poulin, F. J. \& Swaters, G. E., Sub-inertial dynamics of density-driven flows in a continuously stratified fluid on a sloping bottom. I. Model derivation and stability conditions. Proceedings of the Royal Society of London A, 455, pp. 2281-2304, 1999.

[6] Reszka, M. K., Swaters, G. E. \& Sutherland, B. R., Instability of abyssal currents in a continuously stratified ocean with bottom topography. Journal of Physical Oceanography, 32, pp. 3528-3550, 2002.

[7] Swaters, G. E., On the baroclinic instability of cold-core coupled density fronts on sloping continental shelf. Journal of Fluid Mechanics, 224, pp. 361-382, 1991.

[8] Swaters, G. E., Nonlinear stability of intermediate baroclinic flow on a sloping bottom. Proceedings of the Royal Society of London A, 442, pp. 249272, 1993.

[9] Swaters, G. E., Numerical simulations of the baroclinic dynamics of densitydriven coupled fronts and eddies on a sloping bottom. Journal of Geophysical Research, 103, pp. 2945-2961, 1998.

[10] Swaters, G. E., On the meridional flow of source-driven abyssal currents in a stratified basin with topography. Part I. Model development and dynamical characteristics. Journal of Physical Oceanography, 36, pp. 335-355, 2006.

[11] Swaters, G. E., On the meridional flow of source-driven abyssal currents in a stratified basin with topography. Part II. Numerical Simulation. Journal of Physical Oceanography, 36, pp. 356-375, 2006.

[12] Swaters, G. E., Mixed bottom-friction-Kelvin-Helmholtz destabilization of source-driven abyssal overflows in the ocean. Journal of Fluid Mechanics, 626, pp. 33-66, 2009. 